\title{
Ces adolescents qui agressent leur mère !
}

\section{Youth aggressing their mother}

\author{
D. Hélin ${ }^{\mathrm{a}}, \mathrm{V}$. Chevalier ${ }^{\mathrm{a}}, \mathrm{M}$. Born ${ }^{\mathrm{b}}$ \\ ${ }^{a}$ Institution publique de protection de la jeunesse de Fraipont, 113, Sur-le-Bois, 4870 Fraipont, Belgique \\ ${ }^{b}$ Service de psychologie de la délinquance et du développement psycho-social, 3, boulevard du Rectorat, bâtiment B33, 4000 Liège 1, \\ Belgique
}

\section{Résumé}

La violence dirigée contre les parents, méconnue pendant de nombreuses années, est un phénomène aujourd'hui dénoncé par les individus qui la subissent. Confrontés dans notre pratique à diverses formes de violence, cet article a pour objet l'étude de l'agressivité intra-familiale en tant que prédicteur de la capacité de l'adolescent placé en institution publique de protection de la jeunesse à l'analyse critique de ses comportements déviants. L'étude d'un échantillon d'adolescents manifestant de la violence envers leur mère nous indique que cette forme de violence est généralement associée à une délinquance polymorphe. Aussi, ces jeunes proviennent régulièrement de familles matricentriques dans lesquelles la carence paternelle et la précarité maternelle sont présentes. Enfin, l'observation de notre échantillon nous révèle que ces jeunes ont une moindre capacité d'autocritique vis-à-vis de leurs comportements déviants.

Mots clés : Adolescence ; Délinquance ; Violence familiale ; Relation mère-enfant ; Désistance

\begin{abstract}
Violence directed against parents, unrecognized for many years, is a phenomenon today denounced by those individuals who are victims of it. Confronted in our practice by diverse forms of violence, the object of this article is the study of intra-family aggressiveness as a predictor of the capability of the adolescent placed in a Public Institution for the Protection of Youth for the critical analysis of his/her deviant behaviours. The study of a sample of adolescents manifesting violence against their mothers shows us that this form of violence is generally associated with polymorphous delinquency. Besides, these young people regularly come from matrifocal families in which paternal absence and maternal precarity are present. Finally, the observation of our sample reveal that these youth have a lesser capability for self-criticism towards their deviant behaviours.
\end{abstract}

Keywords: Adolescence; Delinquency; Family violence; Mother-child relation; Desistance

Le phénomène de la violence familiale a été principalement étudié à travers l'agression des parents envers leurs enfants ou entre eux. De nombreux articles sont régulièrement publiés dans des revues consacrées spécifiquement à la question. Plus rare est l'étude de la violence familiale dirigée vers les parents. Cette forme de violence inter-individuelle, longtemps ignorée dans la littérature consacrée aux comportements délinquants, suscite honte et culpabilité chez ceux qui y sont confrontés. Aussi, à l'instar des vols intrafamiliaux, le phénomène demeure relativement tabou et probablement plus douloureux à révéler que des faits similaires perpétrés par des individus extérieurs à la famille : l'entourage se montre particulièrement tolérant face à ces comportements agressifs, minimisés dans le but de préserver l'image d'une famille harmonieuse et idéale. Depuis deux décennies pourtant, l'agressivité à l'encontre des parents est venue élargir le spectre des préoccupations des chercheurs dans le domaine de la violence intra-familiale. Dans un premier temps, les recherches ont porté sur la victimisation des parents âgés. Actuellement, la violence des adolescents envers leurs parents est un domaine qui attire l'attention des professionnels. Sa prévalence varie entre 3 et $12 \%$ [3]. Les conséquences de cette nouvelle forme de violence sont généralement bénignes au niveau physique (ecchymoses, griffures), mais plus importantes au niveau psychologique. Pour certains auteurs, la famille dans laquelle l'enfant manifeste de l'agressivité envers ses parents présente une structure particulière [3]. Premièrement, la place occupée par l'enfant au sein de sa famille semble privilégiée. Il est le plus souvent l'aîné ou enfant unique et polarise, par conséquent, l'attention de ses parents. Ensuite, le niveau socio-économique de ces familles semble moyen, voire élevé. Il faut également relever que dans les familles, $60 \%$ de l'un ou l'autre parent manifeste une pathologie psychiatrique. Enfin, la dynamique familiale se caractérise par une relation à la mère à connotation incestueuse, une absence symbolique de la fonction paternelle ainsi qu'une recherche par le jeune des limites et de l'autorité. 
Les lieux de placement sont quotidiennement confrontés à la violence et aux différentes formes qu'elle est susceptible de revêtir. Dans une étude [5], cent adolescents, placés en section fermée dans une institution publique de protection de la jeunesse ont fait l'objet d'une observation. Cette recherche visait à étudier les caractéristiques familiales du jeune, ses perceptions des relations familiales ainsi que leur influence sur la désistance, c'est-à-dire la capacité du jeune à prendre des distances vis-à-vis de ses conduites antisociales. Les résultats indiquent qu'une relation non conflictuelle avec la mère augmente toujours le pronostic de désistance du jeune. Ils suggèrent également qu'une relation sincère et non conflictuelle du jeune entre la mère et son enfant favorise l'adhésion au placement et par conséquent envisage les perspectives d'une meilleure insertion. Ces constatations ont suscité une réflexion quant à l'importance du rôle de la mère dans la problématique de la délinquance. Aussi, constatant l'ampleur de cette nouvelle forme de violence envers la mère, nous avons cherché à étudier la capacité de désistance du jeune adolescent placé lorsque la relation à la mère est empreinte de violence. La présente étude, après en avoir défini les aspects méthodologiques, décrit les caractéristiques de l'échantillon en terme de personnalité de l'adolescent mais aussi en terme de relations familiales et de caractéristiques familiales : le but ultime étant d'examiner la présence d'agressivité envers la mère, signe d'une relation conflictuelle, qui affecterait la capacité du jeune à l'autocritique et à l'introspection.

\section{1. ÉTUDE CLINIQUE}

\subsection{Cadre de la recherche}

Nous avons observé, avec un certain recul, les manifestations de ce nouveau type de comportement agressif, à savoir la violence du jeune envers sa mère, dans une population d'adolescents placés en institution publique de protection de la jeunesse (IPPJ). En Belgique, les IPPJ sont destinées à accueillir des jeunes entre 12 et 18 ans ayant commis des faits qualifiés d'infraction. Le groupe des IPPJ comporte cinq institutions qui poursuivent les objectifs pédagogiques communs suivants : permettre la réinsertion sociale des mineurs, leur donner la possibilité d'acquérir une meilleure image d'eux-mêmes et assurer la protection de la société.

\subsection{Recueil des données}

Les données ont été recueillies à l'aide d'entretiens psychologiques approfondis, d'entretiens centrés, ainsi qu'à l'aide d'entretiens familiaux, réalisés soit à l'institution, soit au domicile familial. L'échelle de mesure de l'adaptation sociale et personnelle pour les adolescents québécois [7,9], a également été utilisée.

Parmi ces outils, l'entretien centré, semi-ouvert, a pour objectif d'amener l'individu à évoquer un thème précis, énoncé par l'intervenant. Ce dernier, contrairement à l'enquête par questionnaire, ne dispose pas d'une grille de question préétablie, mais évoque, sous une forme qu'il est libre de choisir, une série de points précis en relation avec l'événement traité. L'interviewé répond donc aux questions de bases et l'intervenant l'invite à approfondir certains éléments de sa réponse tout en recentrant l'entretien autour du thème lorsque celui-ci s'en éloigne.

\subsection{Caractéristiques générales de notre échantillon}

\subsubsection{Profil général des adolescents}

La population se compose de 16 jeunes, âgés de 14 à 17 ans, issus d'une population de 72 adolescents placés en section fermée à l'IPPJ de Fraipont entre mars 2000 et février 2002 (soit $22 \%$ de la population de l'institution). Ils ont commis des actes de violence à l'égard de leur mère. Tous sont belges, à une exception près, alors que la population générale de l'établissement est largement constituée de jeunes d'origine maghrébine. Les sujets ont fait preuve de violences verbales, physiques ou psychologiques envers leurs mères, de manière suffisamment virulente ou répétitive pour que celles-ci déposent plainte ou qu'elles estiment important pour leur équilibre psychologique ou pour leur sécurité, d'en faire part aux intervenants.

Parmi notre échantillon, l'agression envers la mère n'apparaît, le plus souvent, que comme une conduite inadaptée parmi d'autres, beaucoup plus classiques. En effet, elle n'est que tout à fait exceptionnellement à l'origine du placement. En outre, ces adolescents se caractérisent par une délinquance souvent polymorphe, persistante et d'aggravation progressive.

Les faits, constitués d'infractions contre les biens pour seulement trois d'entre eux et de violences diverses pour les 13 autres, débutent à la période d'adolescence ou de préadolescence. Il s'agit essentiellement de vols divers, de vols de voitures, d'agressions seul ou en bande, d'arrachages de sacs, d'actes de pyromanie (1), d'attentat à la pudeur (1), de viol (1), de consommation et de trafic de stupéfiants (3) ainsi que d'escroquerie (1). 
Le passé de ces adolescents est souvent lourd, chargé d'épreuves pénibles et de souffrances vives. Tous, sauf un, ont connu des placements antérieurs, parfois précoces et souvent multiples. Certains ont cumulé des placements dans le cadre de l'aide à la jeunesse (en institution privée, en institut médico-pédagogique, voire en institution psychiatrique, suite à des problèmes familiaux ou à d'importantes difficultés comportementales) et des placements judiciaires.

Le fonctionnement psychologique de certains de ces jeunes paraît imprégné d'immaturité affective et relationnelle. D'autres présentent une personnalité abandonnique (8/16) et un seul a un profil de personnalité agressive.

\subsubsection{Structure familiale des adolescents}

Tous ces adolescents, ayant commis des violences à rencontre de leur mère, vivent dans des familles matricentriques. Occasionnellement un beau-père ou des beaux-pères apparaissent pour une période plus ou moins longue, plus ou moins heureuse. Un seul couple stable a été véritablement reconstitué. La séparation parentale intervient entre la naissance et l'âge de quatre ans pour dix cas sur seize, entre six et dix ans pour quatre cas, à quinze ans pour un et enfin le dernier a perdu son père à l'âge de six ans.

Tous nos sujets ont donc vécu l'absence de père, et par conséquent de figure d'identification. En effet, nous observons dans notre analyse que les pères des sujets ont presque tous démissionné. Dans deux cas, l'abandon est total. Dans neuf cas, on peut parler d' absence du père : celui-ci est connu et reconnu, il apparaît à l'une ou l'autre occasion mais n'intervient nullement dans l'éducation de son enfant : il manque à l'accueillir les week-ends et les contacts, même occasionnels, font défaut. Dans trois cas, ses interventions sont imprégnées d'attitudes de rejet et pour avoir été sollicitée contre son désir réel, l'expérience s'est soldée par un échec. Le seul père soucieux de son rôle se trouve totalement disqualifié tant au niveau de ses compétences qu'au niveau de son statut parental. Le quinzième, présent de façon illusoire en raison d'une passivité redoutable, ne réintègre le foyer conjugal que lorsque son fils revient en congé. Enfin, le dernier est décédé de mort violente dans un contexte de conflits politiques.

Non seulement ces jeunes souffrent de l'absence du père et de son soutien affectif, économique, social et instrumental mais ils se voient également privés de la compétence parentale qui protège des risques associés à la séparation. Ils sont aussi profondément marqués par cette image négative portée, intentionnellement ou non, par le père.

En ce qui concerne les mères, nous remarquons qu'elles connaissent une vie généralement précarisée, affaiblie par la maladie (6/16), par l'alcoolisme (3/16), par une fragilité nerveuse et émotive les amenant à vivre avec plus ou moins de régularité et d'intensité des épisodes dépressifs (6/16). Nombre d'entre elles souffrent d'une situation socio-économique défavorable. Seules deux mères sur 16 sont engagées dans un emploi régulier. Une troisième, en plus de ses indemnités de chômage, effectue quelques heures de prestation dans l'agence locale pour l'emploi de sa commune. Les 13 autres mères disposent de revenus de remplacement : minimex, chômage, mutuelle, allocation d'invalidité, pension alimentaire. Ces mères connaissent donc, en général, une situation de dépendance sur le plan économique, parfois de longue date.

Outre une situation socio-économique précaire, nous constatons également que les mères de notre échantillon ont été victimes de violences diverses et parfois multiples. En effet, dans $87 \%$ des cas (14/16), elles ont connu dans leur existence de femme, d'épouse, parfois d'enfant, une violence physique (13), parfois sexuelle (2), psychologique (1) ou encore politique (1). Il apparaît donc que ces mères ont, dans une certaine mesure, témoigné d'une difficulté à se protéger de la violence d'autrui et à mettre des limites à leurs agresseurs. Par là, elles ont exprimé à leurs fils leurs difficultés à se faire respecter et à faire entendre leurs droits et besoins. Cette difficulté a d'ailleurs pu compromettre leur capacité à protéger leur enfant des blessures de l'existence, des échecs, des frustrations et de la violence d'autrui. Il semble important de souligner ici que l'enfant a souvent été témoin des violences subies par sa mère. Nous pouvons, par conséquent, faire l'hypothèse que le passage à l'acte du jeune s'en est trouvé facilité. En effet, ces épisodes de violences créent un climat propice à la reproduction, par l'enfant, des stratégies qu'il observe et dont il est la victime indirecte. Par la suite, le jeune peut être amené à adopter ces stratégies comme des modes de transactions acceptables. Par ailleurs, Segond [12] rapporte combien les conflits relationnels, la mésentente, la violence intra-familiale, l'incohérence d'un contrôle parental arbitraire génèrent un climat d'incertitude et d'insécurité permanentes et se retrouvent en force dans les cas de délinquance grave. Dès lors, plus le niveau de conflit entre les parents est élevé plus le risque d'extériorisation agressive est important. Chez 14 de nos 16 mères, ce niveau est très élevé puisque la violence conjugale a fait partie de leur quotidien pendant des périodes plus ou moins longues et a en outre amené à la rupture du couple, de manière 
choisie ou subie.

Des auteurs rapportent, de la collation de diverses études, la force de la relation entre les conflits familiaux (incluant la discorde ainsi que la violence entre les parents, l'exposition des enfants à des épisodes violents) et le comportement violent des enfants [6]. Cette observation a également été décrite par Wolfe et al. [14] selon lesquels le risque de passage à l'acte violent sur la mère par un adolescent est d'autant plus grand que celui-ci a été témoin de violence à l'égard de sa mère par le partenaire de celle-ci.

\subsubsection{Type de violence}

Tout d'abord, nous constatons que la violence des jeunes revêt différentes formes. Comme le précise Le Blanc [10], il convient de distinguer cinq types d'utilisation de la force dans le cadre des relations interindividuelles : les agressions symboliques, les agressions verbales, les agressions psychologiques, les agressions physiques et les agressions matérielles.

Ces divers types de violence se retrouvent dans notre échantillon, souvent de manière combinée. Cependant, nous nous sommes centrés essentiellement sur les agressions verbales - d'une part, parce qu'elles n'échappent à aucun de nos sujets et d'autre part, parce qu'elles peuvent recouvrir également les agressions de type

psychologique lorsque leur contenu est coloré de menaces, de chantage, d'intimidation et de dénigrement — ainsi que sur les agressions physiques. Quant aux agressions matérielles, fréquentes dans les milieux déjà matériellement défavorisés, elles apparaissent souvent comme la partie visible de l'iceberg, tant pour la mère qui peut s'en servir comme un relais pour aborder la violence plus grave dont elle a été victime, que pour l'adolescent qui peut tenter de minimiser son agressivité derrière ces actes d'apparence moins grave. Entrent également dans cette catégorie de violence matérielle les vols intra-familiaux.

Si les 16 adolescents recourent à la violence verbale, tous ne passent pas à l'acte physiquement. C'est néanmoins le cas dans neuf situations. Les actes agressifs et violents dirigés contre la mère comportent aussi bien des bousculades, plus ou moins violentes, que des tentatives d'étranglement ou des menaces au couteau.

Par le recours régulier aux menaces verbales, aux injures et au chantage (ainsi qu'à la fugue, la délinquance, la violence), le jeune place sa mère face à un choix douloureux alors que celle-ci est déjà en peine de fournir fermeté et cohérence éducative et est en proie à ses angoisses et à la reviviscence de ses souvenirs de violence subie. En effet, ces mères sont souvent perçues comme des femmes soumises, anxieuses, faibles ou dépressives. Mais elles sont également considérées comme des personnes ambivalentes, partagées entre la fusion et la surprotection d'une part, et le rejet de leur enfant d'autre part, lui imposant des variations de distance intenses et déstructurantes. Ainsi, ces adolescents voient tour-à-tour leur mère accepter d'une certaine manière leurs comportements déviants et les protéger des interventions de l'environnement extérieur, pour ensuite essuyer leur rejet et accuser une mise à distance perçue et vécue par certains comme un abandon. Ce sont effectivement ces jeux, comme le précise Segond [12], fondés sur des mouvements relationnels cycliques et contradictoires extrêmement nocifs, qui sont à l'origine des structures abandonniques. D'autres mères, davantage inscrites dans la dépendance et dans des modèles d'assistance, témoignent à l'égard de leur garçon de passivité et de laxisme. Repliées sur elles-mêmes, elles accèdent peu au soutien extérieur et ne manifestent que peu de démarches actives pour intervenir à l'égard de leurs enfants. Dès lors, les relations mère-fils se voient régulièrement dominées par un sentiment de toute puissance, voire d'omnipotence du fils à l'égard de sa mère (7/16). Il faut craindre, dans le chef de ces adolescents, une capacité d'empathie des plus ténues et une aptitude relationnelle des plus pauvres, ou à tout le moins marquée par leur égocentrisme et la jouissance du pouvoir exercé sur autrui.

\subsection{Conclusion et discussion}

L'analyse des caractéristiques socio-économique des jeunes de notre échantillon nous apprend qu'ils sont issus de familles vivant dans des conditions précaires voire défavorisées. La majorité des mères de notre échantillon bénéficie de revenus de remplacement : elles se trouvent par conséquent dans une situation de dépendance économique. Ces données contrastent avec les résultats obtenus par Dugas, Mouren \& Halfon [3], qui constatent à partir de leur population tout venant, que l'origine socio-économique des jeunes de leur échantillon est globalement moyenne voire élevée. Cette réalité observée dans notre échantillon témoigne notamment d'une difficulté maternelle à identifier ses compétences et à les faire valoir de manière à se créer une place sur le marché de l'emploi pour prendre part à la société active. Dès lors, il apparaît malaisé pour ces femmes de soutenir leur fils dans une scolarité épanouissante, de les aider à traverser les difficultés qui y sont associées et peut-être aussi de les amener à intégrer des valeurs de travail et d'intégration socioprofessionnelle. Par ailleurs, Gavray [5] défend que l'insertion professionnelle de la mère lui permettrait d'améliorer la situation de son enfant, 
rendant celle-ci plus favorable à l'expression de valeurs liées à la famille. Meilleures capacités communicationnelles, stratégies de résolution de problème plus efficaces, présence active auprès des enfants, autonomie accordée mais sous contrôle, incitation à la créativité, plus grande surveillance du temps passé devant la télévision (et donc aussi de l'exposition à des modèles de violence) sont autant d'éléments amenant les mères qui travaillent à être de meilleures alliées et interlocutrices pour leurs enfants que ne le sont les mères qui ne travaillent pas à l'extérieur.

L'étude du noyau familial des adolescents de notre échantillon nous révèle une structure matricentrique dans laquelle les pères ou substituts paternels semblent avoir une moindre place. Cette carence de la fonction paternelle parait caractéristique des familles imprégnées d'un climat de violence dirigée vers la mère [3]. Or, on sait aujourd'hui combien la présence d'un modèle d'identification masculine est primordiale. Son absence peut se révéler nuisible à l'évolution de l'adolescent aux prises avec un problème d'identité. De plus, il est fréquent de constater que la relation troublée père-fils occasionne chez l'adolescent d'énormes difficultés à intérioriser les règles et à affirmer sa capacité d'autocontrôle [1]. Elle le rend aussi plus vulnérable aux influences négatives et le pousse à adopter des comportements à caractère compensatoire destinés à satisfaire son besoin d'affirmation. Dans la seconde moitié de l'adolescence, l'importance du père prédomine. Au cours de cette phase de développement, l'adolescent aurait un besoin accru de ce parent du même sexe pour consolider ses acquis, construire et façonner son identité [4]. Or, dans notre échantillon, le jeune est confronté à une absence totale de figure paternelle, absence d'autant plus grande que ces mères n'ont pas voulu ou ne sont pas parvenues à offrir une image masculine de remplacement fiable et adéquate.

L'importance du rôle du père se manifeste également à d'autres niveaux. Parlse (1980), cité par Le Blanc [10], met l'accent sur son influence majeure dans l'enracinement de la délinquance. Les situations de nos adolescents sont saturées d'indicateurs actifs concernant l'impact du rôle paternel, faisant ici défaut : compréhension du père, connaissance, intérêts et contrôle des activités du fils par le père, connaissance des amis, attitude agréable du père, amusements communs, disponibilité, discussion père-fils, gestes d'affection, vacances communes, entraide, punition adéquate par le père.

Cyrulnik [2] met lui aussi en évidence les conséquences d'une carence paternelle au niveau des relations à la mère et du développement de l'enfant. Une triade déficitaire entre l'enfant, le père et la mère, amène la mise en place d'une « relation d'emprise, délicieuse d'abord puis gavante jusqu'à la nausée plus tard ». Les garçons s'identifiant à leur mère doivent la quitter un jour sous peine d'éprouver des angoisses incestueuses. S'ils ne disposent pas de tuteurs de développement pour les aider à ce départ, ils ne trouveront pas d'autres solutions que l'inhibition ou l'explosion.

La dimension incestueuse de la relation mère-fils dans les familles caractérisées par cette nouvelle forme de violence intra-familiale est également relevée par Dugas, Mouren \& Halfon [3]. En effet, ces derniers soulignent la difficulté des mères à céder autonomie et dépendance à leur enfant, les enfermant ainsi dans une relation exclusive et infantilisante. Le passage à l'acte agressif représente donc une tentative de lui échapper. Segond [12], quant à lui, observant que des comportements de violence à l'égard de l'un des parents est une situation fréquente dans les familles monoparentales, constate qu'elle peut être l'expression d'une résistance contre un risque d'emprise sexualisée (sans inceste réalisé) du parent du sexe opposé. Les adolescents, exposés à une situation angoissante de confusion des rôles, ont donc recours à la violence pour mettre de la distance et faire cesser une relation devenue trop fusionnelle avec la mère. Enfin, une étude de Shiff, rapportée par Cyrulnik [2], sur un groupe d'enfants sans père constate que ces enfants, trop attachés à leur mère, se montrent plus dépendants, moins explorateurs, plus émotifs et plus conformistes se soumettant à une mère qu'ils agressent cependant en cas de frustration. La mère et le fils, dit Huerre [8], peuvent se disputer la barre dans un face-à-face empli de violence incestueuse. Nous observerons dans notre échantillon que cette situation est fréquente puisque six de nos mères ont développé une relation fusionnelle avec leur adolescent. Outre la composante fusionnelle de la relation mère-fils, Maqueda [11] évoque, chez ces mères, une remarquable tolérance à l'égard de la tyrannie que leurs enfants exercent sur elles par l'imposition d'ordres et de rituels.

La perturbation de l'autorité parentale constitue également une caractéristique des familles subissant la violence de leur enfant [3]. En effet, les chefs de famille monoparentale affirment moins activement leur autorité sur leur adolescent : ils sont plus permissifs et plus négligents. La responsabilité qu'un parent unique doit assumer, pesante, difficile, répétitive, dans tous les secteurs de la vie quotidienne, tend à l'affaiblissement du rôle parental. Tantôt simplement pour se soulager lui, tantôt parce qu'il doute de ses propres capacités, le parent unique manifeste moins activement sa fonction éducative, protectrice et relationnelle ou tend à démissionner laissant le jeune, trop et trop tôt, livré à lui-même. 
L'objectif principal de notre étude est de réfléchir à la capacité de l'adolescent placé à poser un regard critique sur son passage à l'acte agressif (plus particulièrement envers sa mère). En d'autres termes, nous nous interrogeons sur sa capacité d'autocritique et sa capacité d'introspection.

Les données de notre étude nous indiquent en premier lieu que certaines caractéristiques familiales et personnelles apparaissent dominantes : la monoparentalité, le vécu antérieur de violences de la mère, sa fragilité sur le plan psychologique mais aussi social et la conjonction avec des personnalités adolescentes de type abandonnique, carence affectif ou de toute puissance se dégagent de l'analyse de notre échantillon et de nos tentatives de compréhension de cette forme de dysfonctionnement. En ce qui concerne notre hypothèse principale, relative à la capacité d'autocritique et d'introspection, nous relevons qu'une majorité d'entre eux n'accède nullement à la remise en cause de soi et n'est pas encline au travail d'introspection. Les adolescents chez qui cette capacité fait défaut sont ceux qui manifestent la violence intra-familiale la plus importante. En effet, ils cumulent les différentes formes de violences (verbale, psychologique et physique) et s'inscrivent quotidiennement dans un jeu relationnel emprunt de toute puissance à l'égard de leur mère.

En corollaire, un faible nombre d'adolescents ayant accès à la réflexion et à l'introspection expriment une violence essentiellement verbale et présentent un profil de personnalité abandonnique ou dépressif.

Cette étude nous a également permis de constater que cette nouvelle forme de délinquance n'est pas réellement dissociée d'un autre type de délinquance : ce n'est qu'exceptionnellement qu'elle justifie le placement dans les établissements public de protection de la jeunesse. Est-ce à dire que tous les jeunes qui manifestent de la violence envers leurs mères sont avant tout des auteurs d'actes plus généralement délictueux ? Ou est-ce plutôt que cette violence sur la mère manque d'être véritablement reconnue comme telle, banalisée et fortement étouffée?

Ces constatations sont-elles suffisantes pour amener la conclusion selon laquelle seul le second type d'adolescents accède à la souffrance et à la douleur de son vécu ? Cette conclusion nous amènerait-elle également à l'idée que les premiers, à l'inverse des seconds, sont coupés de leurs affects et évoluent dans la révolte et la colère aussi bien au niveau social que familial, ayant pour seul interlocuteur leur mère ? Pourrait-on enfin prédire, grâce à la capacité d'introspection et d'autocritique (afin de briser un cercle que l'on devine vicieux), ce type de délinquance interpersonnelle?

Du fait de cette coexistence de l'agressivité envers la mère avec d'autres conduites déviantes, il est d'autant plus ardu de mettre en relief des facteurs de risque propres à ce type de délinquance. Dans ce domaine comme dans d'autres, ainsi que l'explique Vitaro [13], le nombre de prédicteurs de risque, plus que leur nature particulière, constitue la clé d'une prédiction optimale. Mais il est difficile de départager l'apport spécifique de chaque variable, d'autant que l'on ne peut ignorer le cumul des facteurs de risque mais aussi leur interaction, leur relativité ou encore la période de développement de celle-ci.

\section{Références}

[1] Born M. Jeunes déviants ou délinquants juvéniles. Bruxelles: Mardaga; 1987.

[2] Cyrulnik B. Les vilains petits canards. Paris: Odile Jacob; 2001.

[3] Dugas M, Mouren MC, Halfon O. Les parents battus et leurs enfants. Psychiatr Enfant 1985;28:185-219.

[4] Frechette M, LeBlanc M. Délinquance et délinquants. Montréal: Gaëtan Morin; 1987.

[5] Gavray C. Conduites juvéniles contemporaines et transformations socio-familiales. In: Born M, Thys P, editors. Délinquance juvénile et famille. Paris: L'Harmattan; 2001.

[6] Hawkins JD, Herrenkhol T, Farrington DP, Brewer D, Catalano RF, Harachi TW. A review of predictors of youth violence. In: Loeber R, Farrington DP, editors. Serious and violent juvenile offenders: risk factors and successful interventions. Thousand Oaks, Californie: Sage Publications; 1998. p. 106-46.

[7] Helin D, Born M, Chevalier V. L'évolution des jeunes délinquants en lien avec l'adhésion à un projet et la communication intra-familiale. In: Born M, Thys P, editors. Délinquance juvénile et famille. Paris: L'Harmattan; 2001

[8] Huerre P. L'adolescence en héritage. Paris: Calmann Lévy; 1996. 
Published in: Neuropsychatrie de l'enfance et de l'adolescence (2004), vol. 57 Status : Postprint (Author's version)

[9] Le Blanc M. Maspaq, mesure de l'adaptation sociale et personnelle pour les adolescents québécois : manuel et guide d'utilisation. $3^{\mathrm{e}}$ édition. Montréal: université de Montréal, groupe de recherche sur les adolescents en difficulté; 1996.

[10] Le Blanc M. Les comportements violents des adolescents. In: Cusson M, Ouimet M, Proulx J, editors. Les violences criminelles. Sainte Foy, Québec: Presses de l'université Laval; 1999.

[11] Maqueda AF. Coups de fil pour coups de fils, rencontre dans une permanence avec des mères battues par leurs enfants adolescents. Inf Psychiatr 1988;64:907-15.

[12] Segond P. La dimension familiale dans la délinquance des adolescents. Bull Psychol 1999;52:443.

[13] Vitaro F, Dobkin PL, Gagnon C, LeBlanc M. Les problèmes d'adaptation psychosociale chez l'enfant et l'adolescent : prévalence, déterminants et prévention. Montréal: Presses de l'université du Québec; 1994.

[14] Wolfe DA, Jaffe P, Wilson SK, Zak L. Children of battered women: the relation of child behaviour to family violence and maternal stress. J Consult Clin Psychol 1985;53:657-65. 